\title{
Experimental Study of the Characteristics of the Flow in the First Rows of Tube Banks
}

\author{
Cláudio R. Olinto ${ }^{1)}$, Luiz Augusto Magalhães Endres ${ }^{2)}$ and Sergio Viçosa Möller ${ }^{3)}$ \\ 1) Federal University of Rio Grande do Sul - PROMEC, Porto Alegre, Brazil (dfscro@ furg.br ) \\ 2) Federal University of Rio Grande do Sul - IPH, Porto Alegre, Brazil (endres@ufrgs.br) \\ 3) Federal University of Rio Grande do Sul - PROMEC, Porto Alegre, Brazil (svmoller@ufrgs.br)
}

\begin{abstract}
In the present work, the flow through tube banks is studied in order to understand the random and transient behavior of the instabilities and phenomena that occur in the first rows of tube banks and propagates to its interior. The study is performed using hot wire anemometry in an aerodynamic channel and flow visualizations in a water channel. Three tube banks with square arrangement and pitch-to-diameter ratios of 1.26, 1.4 and 1.6 were studied. The Reynolds number range for the velocities measurements, computed with the tube diameter and the flow velocity in the narrow gap between tubes, was $7-8 \times 10^{4}$. Continuous and discrete wavelets analyses were applied to decompose the velocity results, thus allowing the analysis of phenomena in a time-frequency domain. Visualizations in a water channel were made, using a dye injection directly in the flow, in order to help the understanding about the phenomena found. A tube bank with a square arrangement and 1.26 pitch-to-diameter ratio was used, and the experiments were performed in a Reynolds number range of $3-4 \times 10^{4}$. The main results show the presence of instabilities generated from the second row of the tube bank, which propagates to the interior of the bank, generating a behavior where the three orthogonal components of the flow are equally important. The three-dimensional behavior of the flow is responsible for a redistribution of mass inside of the bank of tubes that leads to velocity values and Strouhal numbers not expected for the studied geometry.
\end{abstract}

\section{INTRODUCTION}

Flow through cylinder clusters are found in several engineering applications in nuclear and process industry: in tube banks of heat exchangers, offshore platform, chimney groups, stakes of bridges and piers are example of this use. The flow through solid structures is responsible for dynamic loads and may produce vibration on the structures leading to fatigue crack and fretting-wear damage of the components. The kinds of cross-flow distinguish as a function of cylinder arrangements. In tube banks with large distance between center lines (pitch), the dynamic loads are mainly associated with the vortex shedding process, with defined frequencies. In tube arrangement with small aspect ratio (pitch-to-diameter ratio), the turbulent flow is characterized as broad band turbulence, without a defined shedding frequency [1]. Zukauskas studied the flow through tube banks with in-line arrays and compare this flow with a straight channel, being the velocity distribution strongly influenced by the velocity in the narrow gaps [2].

Indrusiak et al. [3] applied wavelet transforms to study the turbulent flow through tube banks. They analyzed the behavior on the wake region of a tube in the bank and found that the mode shift of the flow in both sides like a bistable flow. Olinto et al. [4] confirmed these results and evidenced the presence of a nonstationary phenomenon characterized by the alternation in the magnitude of de mean velocity measured behind the third row. In this work, they introduced the visualizations in a water channel for help the interpretation of the results. In another work, Olinto et al. [5] performed an experimental study in order to compare the statistic characteristics of bistable flow through two cylinders placed side-by-side with the flow in tube bank. A compressive analysis using spectral tools and visualizations for several tube bank geometries is presented in [6], where the classical results are summarized.

\section{EXPERIMENTAL TECHNIQUE}

\section{Aerodynamic channel}

The test section is the same used in [7], being a $2320 \mathrm{~mm}$ long rectangular channel, with $146 \mathrm{~mm}$ height and a maximal width of $193 \mathrm{~mm}$, Fig. (1). Air, at room temperature, is the working fluid, driven by a centrifugal blower, passed by a diffuser and a set of honeycombs and screens, before reaching measurement location with about $1 \%$ turbulence intensity. After the screens, a Pitot tube was placed at a fixed position to measure the reference velocity for the experiments and for the calculation of the Reynolds number at the steady state condition.

For measurements of velocity and velocity fluctuations, DANTEC constant temperature hot wire anemometers were applied. A double hot wire probe was applied. It has one wire perpendicular to the main flow and a second wire inclined $45^{\circ}$ to the main flow, so that two components of the velocity vector and their fluctuations could be measured simultaneously.

The tube banks investigated had a square arrangement and pitch-to-diameter ratios (P/D) of 1.26, 1.4 and 1.6. The tubes had $32 \mathrm{~mm}$ of diameter. The flow was perpendicular to tube axes. 


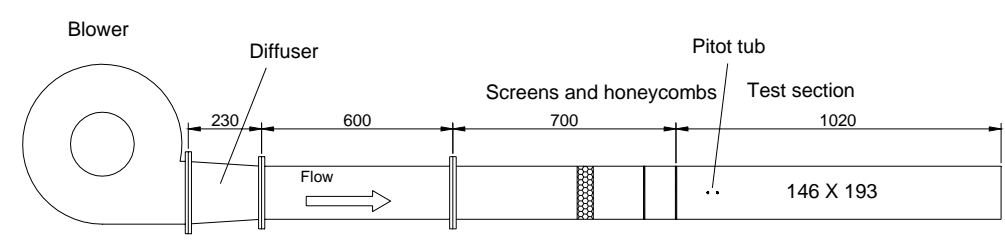

Figure 1. Schematic view of the aerodynamic channel.

\section{Water channel}

The water tests were performed in a closed-circuit water channel facility of the Hydraulic Research Institute IPH/ UFRGS. The tubes were fixed vertically in the translucent plexiglas test-section, where the top wall also had the function of avoiding the effect of free surface oscillations. A flood-gate and a gate valve were used to control respectively the water level and the flow rate. The bank was made from commercial PVC tubes with diameter of 75 $\mathrm{mm}$, with exception of the central one which was handmade from a translucent plexiglas plate (Fig. 2). A mirror was fixed inside this tube at the mid height of the tube, inclined $45^{\circ}$ with the tube axis direction. This arrangement allows the viewing of the flow inside the tube bank of tubes. A hole was drilled at the wall of the tube neighboring the plexiglas tube, in the same row. The dye is gravity forced through this hole by means of an injection needle. The tube bank used for the visualizations had a square arrangement and a pitch-to-diameter ratio (P/D) of 1.26.

Dye injection technique was used to visualize the flow structure in the arrays. Two injection points where used: one upstream to the tube bank and another on the wall of upstream side to the center tube. The images of the flow field were recorded by a video system. Photographs presented in this paper were taken from the videos. Flow visualizations were performed for Reynolds numbers from $3 \times 10^{4}$ to $4 \times 10^{4}$, computed with the tube diameter and the mean channel velocity (incident velocity).

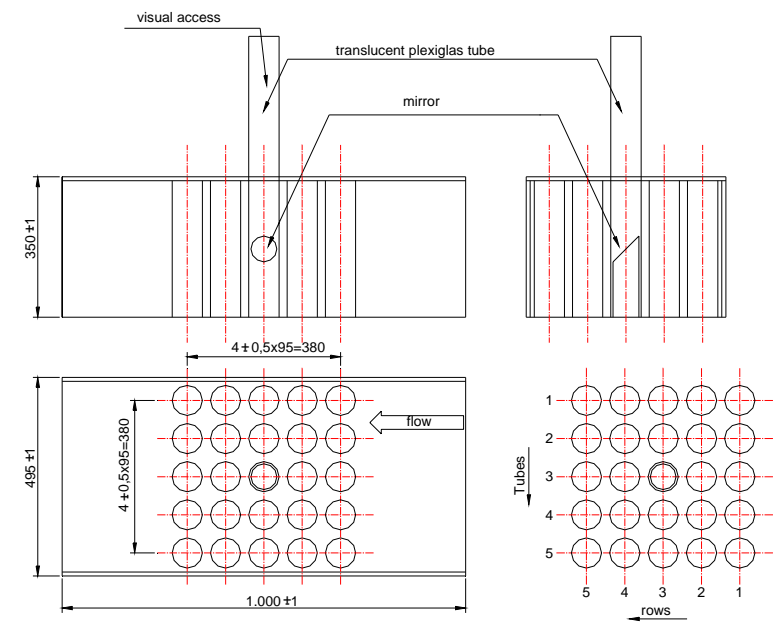

Figure 2. Dimensional scheme of the test section for the flow visualization experiments ( $\mathrm{P} / \mathrm{D}=1.26)$.

\section{MATHEMATICAL TOOLS}

The velocity signals measured inside the tube bank were analyzed using wavelet transforms in order to obtain information about the distribution of the energy of the transient flow over time-frequency domain. It was used the continuous wavelet transform to construct the continuous wavelet spectrum and the discrete wavelet transform in order to decompose the sign measured in wavelet approximations divided in frequency bands. In [5] is described the use of this technique. The mathematic tools was developed using the Matlab® 5.3 software.

\section{RESULTS}

\section{Velocity measurements}

In order to found the transversal flow components, velocity measurements were made using a double wire probe at several places inside of the tube banks. The probe used have a perpendicular wire and an inclined wire related with the own axis. This geometry allow to measure the velocity in the main flow direction and the angle $(\alpha)$ formed between this velocity vector and the channel axis. To found the best locations for the measurements, the visualizations in water channel was used. 
Figure 3 shows the velocity measurement result for the tube bank with $\mathrm{P} / \mathrm{D}=1.26$. The graphics present the velocity modulus and the angle $\alpha$. The flow is in steady state, and the probe is placed behind the central tube of the third row. The Reynolds number, calculated with the gap velocity and the tube diameter was $8.4 \times 10^{4}$ and the sample frequency was $3 \mathrm{kHz}$. The angle is measured in a vertical plane, regarding positive values when the incident velocity on the probe is upwards. As illustrated in Fig.3-a, the only information possible to obtain is the flow turbulent nature.

Figure 3-b shows the interval from $3 \mathrm{~s}$ to $5 \mathrm{~s}$, where some patterns in the flow occur and remain during some time, changing, then, to a new pattern. In the quoted case, from $4.2 \mathrm{~s}$ to $4.5 \mathrm{~s}$, there is a reduction in the velocity fluctuation and the mean value is about $30 \mathrm{~m} / \mathrm{s}$. In the same time interval, the incident angle fluctuation decreases and the mean value is about $20^{\circ}$.
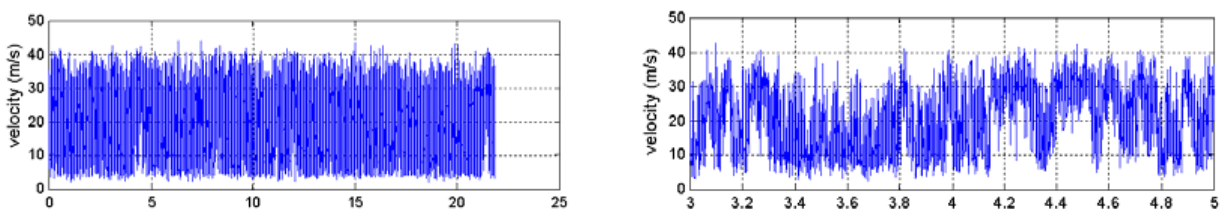

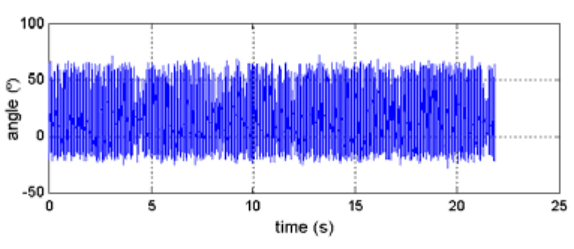

(a)

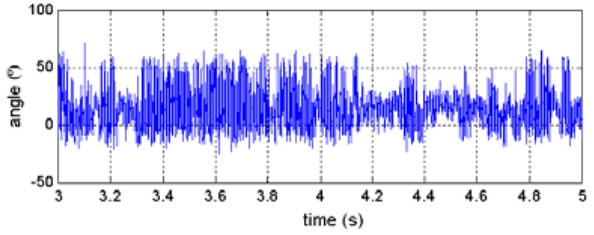

(b)

Figure 3. (a)Velocity and incident angle measured behind the central tube of the third row (P/D = 1.26); (b) Interval from $3 \mathrm{~s}$ to $5 \mathrm{~s}$.

Applying the wavelet discrete transform in order to separate the velocity and angle signals in frequency band, it is possible to better observe these characteristics. Figure 4 shows the decomposition of velocity and incident angle in six frequency bands. The mean velocity value can be observed in the first approximation, until $2.9 \mathrm{~Hz}$. In the interval between $4.2 \mathrm{~s}$ and $4.6 \mathrm{~s}$, the mean velocity value is around $25 \mathrm{~m} / \mathrm{s}$, being modulated by a frequency between $2.9 \mathrm{~Hz}$ and $5.8 \mathrm{~Hz}$ that cause the fall in the instantaneous velocity around the time $4.55 \mathrm{~s}$. At the interval between $3.5 \mathrm{~s}$ and $3.8 \mathrm{~s}$ the velocity stay around $15 \mathrm{~m} / \mathrm{s}$. When the velocity values increase, the higher frequency bands have lower amplitude. On the other side, when the velocity values are reduced, the higher frequencies bands have higher amplitudes. The incident angle changes in association with the velocity values. When the velocity has a high value, the incident angle presents a low value, and in the higher frequency bands the fluctuations are the lowest, showing that, when the velocity is increased, the incident angle undergo a aligning in a defined direction. For lower velocities the angle fluctuations are larger for all frequencies.

In order to analyze the behavior of energy associated with the frequency bands for the velocity signal along time, the spectrogram, calculated through wavelet continuous transform, was constructed. Figure 5-a shows the spectrogram of signal, presented in Fig. 3, where it can be observed that, when the velocity fluctuates around the higher values, there is a larger energy concentration for the lower frequencies ( 3 to $3.3 \mathrm{~s}$ and 4.2 to $4.6 \mathrm{~s}$ ). On the other hand, in the interval where the velocity fluctuates around the lower velocity values, there is an energy spread on several frequencies (3.4 to $4.2 \mathrm{~s}$ ). The incident angle spectrogram (Fig. 5-b) presents an opposite behavior. For the higher velocity values, the incident angle has the lower fluctuation energy for all frequency bands ( 4.2 to $4.6 \mathrm{~s}$.

The second study was made for the tube bank with P/D-ratio of 1.4. The experiment used a sample frequency of $3 \mathrm{kHz}$ and the Reynolds number was $7.4 \times 10^{4}$. Figure 6 shows the instantaneous velocity and incident angle signals. The characteristics presented in this case are similar to that found for the tube bank with $\mathrm{P} / \mathrm{D}=1.26$. It is possible to see that when the velocity increases the fluctuation of incident angle decrease (e.g. 6.5 to $8 \mathrm{~s}$ and $12 \mathrm{a} 13 \mathrm{~s}$ ).

The decomposition in frequency bands, likewise the tube bank with $\mathrm{P} / \mathrm{D}=1.26$, showed that when the velocity increases ( 6.5 to $8 \mathrm{~s}$ and 12 to $13 \mathrm{~s}$, for instance) the velocity fluctuation in higher frequency bands decreases, except for the 23.4 to $46.8 \mathrm{~Hz}$ interval, where the fluctuation is fairly equal during all time studied. The frequency band decomposition for the incident angle shows a similar behavior for the $\mathrm{P} / \mathrm{D}=1.26$ bank. When the velocity has higher values, the flow incidence angle remains at lower values, with lower energy fluctuations. In this geometry, the shift in flow mode is more difficult to observe, because it occurs at shorter time intervals. The times with higher velocity are around $1 \mathrm{~s}$ and the falls in velocity value occur almost as pulses. Thereby for tube bank with $\mathrm{P} / \mathrm{D}=1.4$, the shift among the flow modes does not occurs into characteristic time interval, nevertheless the fluctuation in velocity vertical component can be observed. 

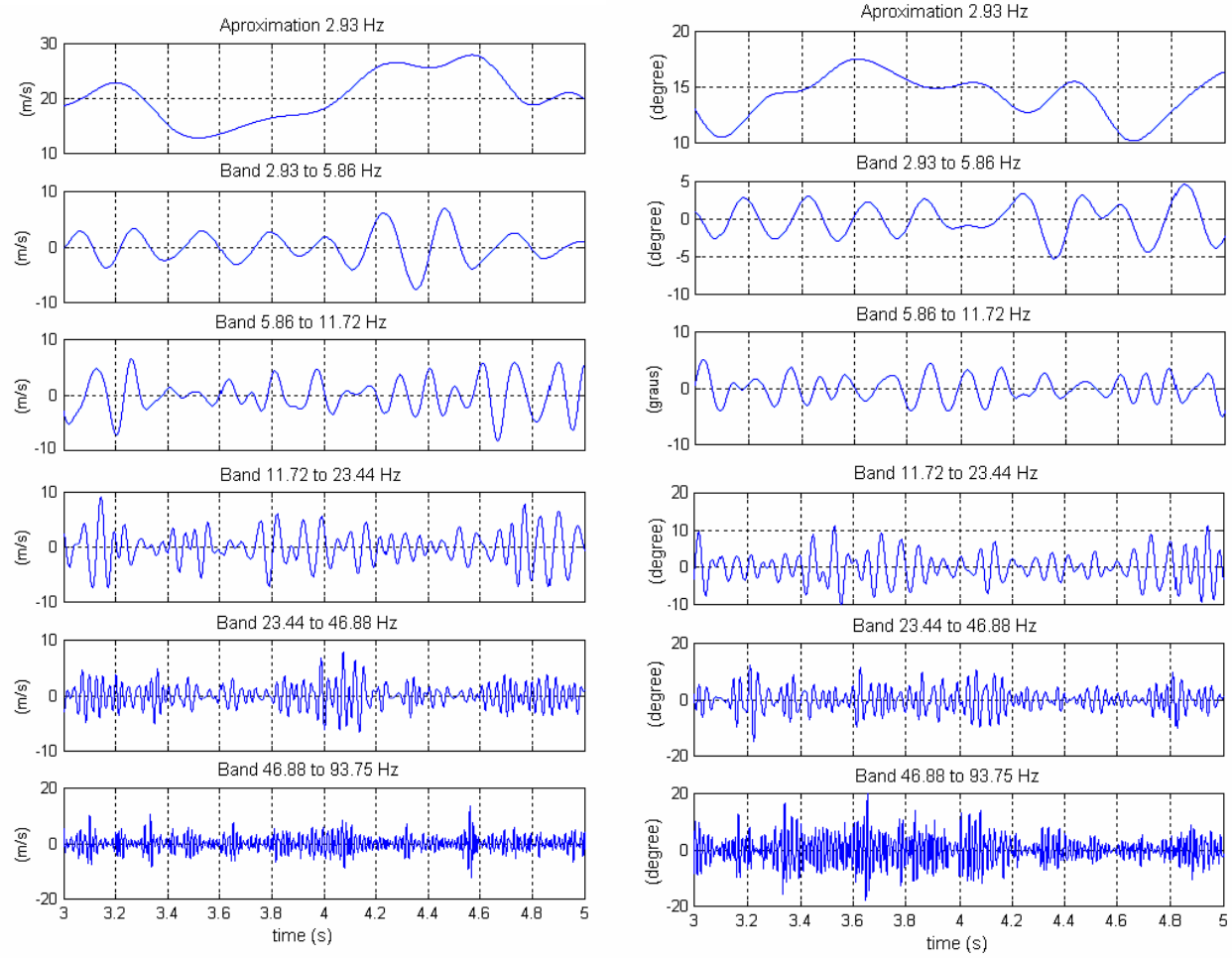

(a)

(b)

Figure 4. (a) Velocity decomposition and (b) incident angle of in Fig.(6) in frequency bands, using wavelet discrete transform. Tube bank with $\mathrm{P} / \mathrm{D}=1.26$.
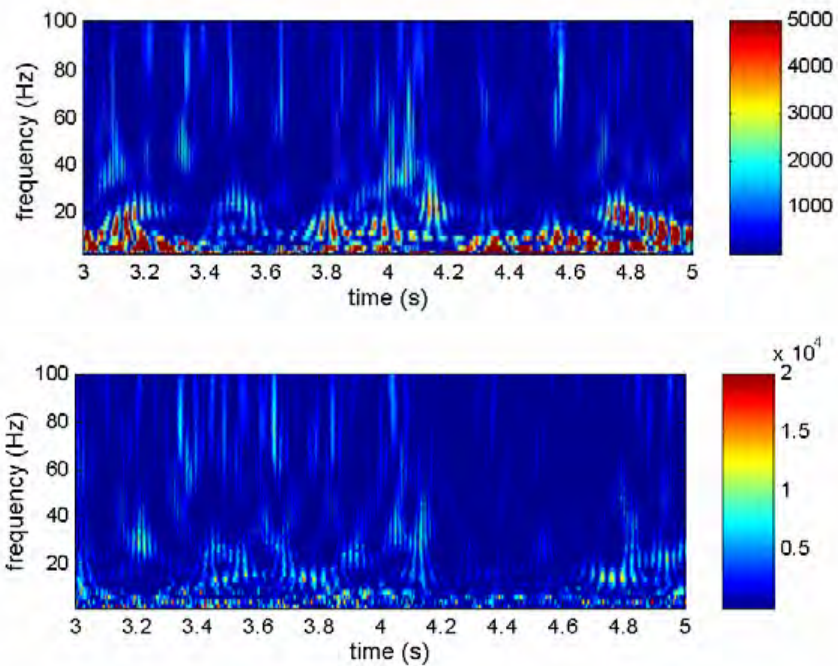

(a)

(b)

Figure 5. (a)Velocity spectrogram for the signal presented in Fig.3 (arbitrary energy color scale); (b) Velocity incident angle spectrogram for the signal presented in Fig.3 (arbitrary energy color scale). Tube bank with P/D=1.26.

For the tube bank with P/D-ratio equal to 1.6, the velocity and incident angle series were obtained behind the third row. The signal is obtained with sample frequency of $3 \mathrm{kHz}$, in steady state flow, with Reynolds number of $7.4 \times 10^{4}$. The simultaneous signals are show in Fig.7. Figures 8-a and 8-b show the velocity and incident angle decomposition in frequency bands. In this series, it is possible to identify the presence of two different flow modes, that occur seven times, from observing directly the instantaneous velocity and incident angle signals. In the velocity signal decomposition (Fig. 8-a) one can observe that, associated to the interval, where the velocity stays at higher values $(\sim 28 \mathrm{~m} / \mathrm{s})$, the fluctuation amplitude, for all frequency bands, has the lower values. On the other hand, when the velocity fluctuates around the lower values $(\sim 17 \mathrm{~m} / \mathrm{s})$, the larger frequency fluctuations are present. In the 
incident angle decomposition a similar behavior is found (Fig. 8-b). During the intervals where the mean angle shows a trend of an alignment at $11^{\circ}$, a reduction in fluctuation amplitude, for all frequency bands is noticed.

In order to analyze more detailed what occurs when the flow mode shift, it is shown the spectrogram from the wavelet continuous transform for the interval from $44 \mathrm{~s}$ to $76 \mathrm{~s}$ (Fig. 9). This interval contains the time when the flow mode shifts ( 60s). Before $60 \mathrm{~s}$ the spectrogram energy is spread for all frequencies. After this time, the spectrograms present lower energy for all frequencies confirming the lower fluctuations at higher velocities.

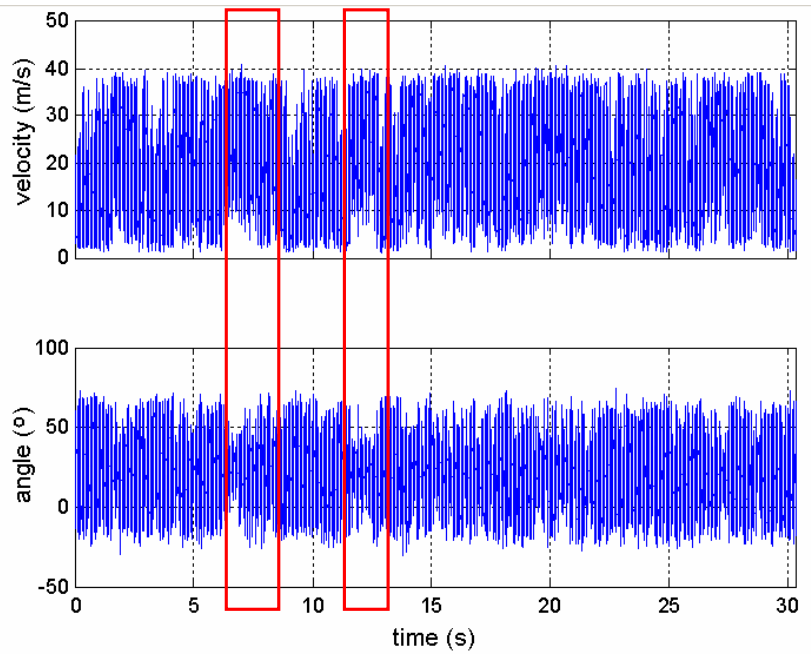

Figure 6. Velocity and incident angle measured behind the center tube at third row $(\mathrm{P} / \mathrm{D}=1.4)$.
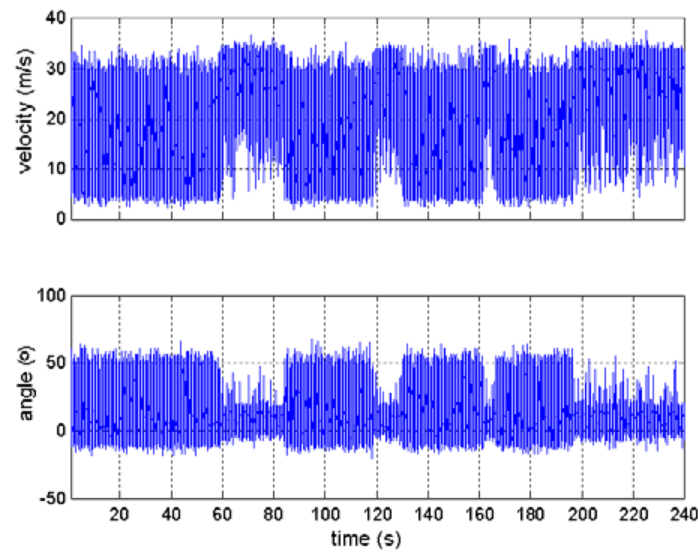

Figure 7. Velocity and incident angle measured behind third row for tube bank with $\mathrm{P} / \mathrm{D}=1.6$.

\section{Visualizations}

The flow visualizations were performed using a tube bank with a pitch ratio of 1.26 . The objective was to provide additional information about the flow behavior when it crosses the first rows of a tube bank. It was made for several Reynolds number, calculated with the mean gap velocities and the tube diameter. The dye injection was made in three ways: using a needle cluster with one needle for each gap - this injection is made $60 \mathrm{~mm}$ below the test section upper wall; through a needle positioned in the center tube wall at its middle height, and finally, using a mobile needle placed upstream of the tube bank and $60 \mathrm{~mm}$ above the channel bottom.

In the experiment shown in Figure 10, the flow close to the test section upper wall undergoes a deviation to the left (from upstream to downstream) and the dye thread, seen through the mirror placed in center tube, has a downward trend. (Fig.10-a). Close to the bottom the flow deviates to the right (Fig.10-b).

Figure 11 presents an opposite flow pattern. The flow close to the upper wall occurs with a deviation to the right whereas the bottom flow deviates to the left. In the mirror, an upward flow deviation, in vertical plane, is now observed. 

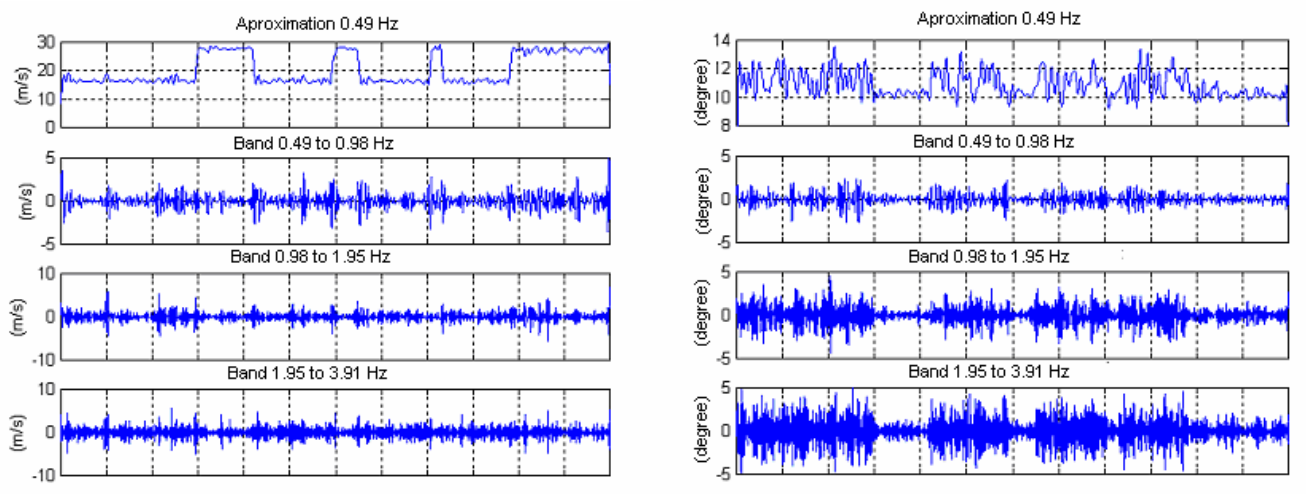

Band 3.91 to $7.81 \mathrm{~Hz}$
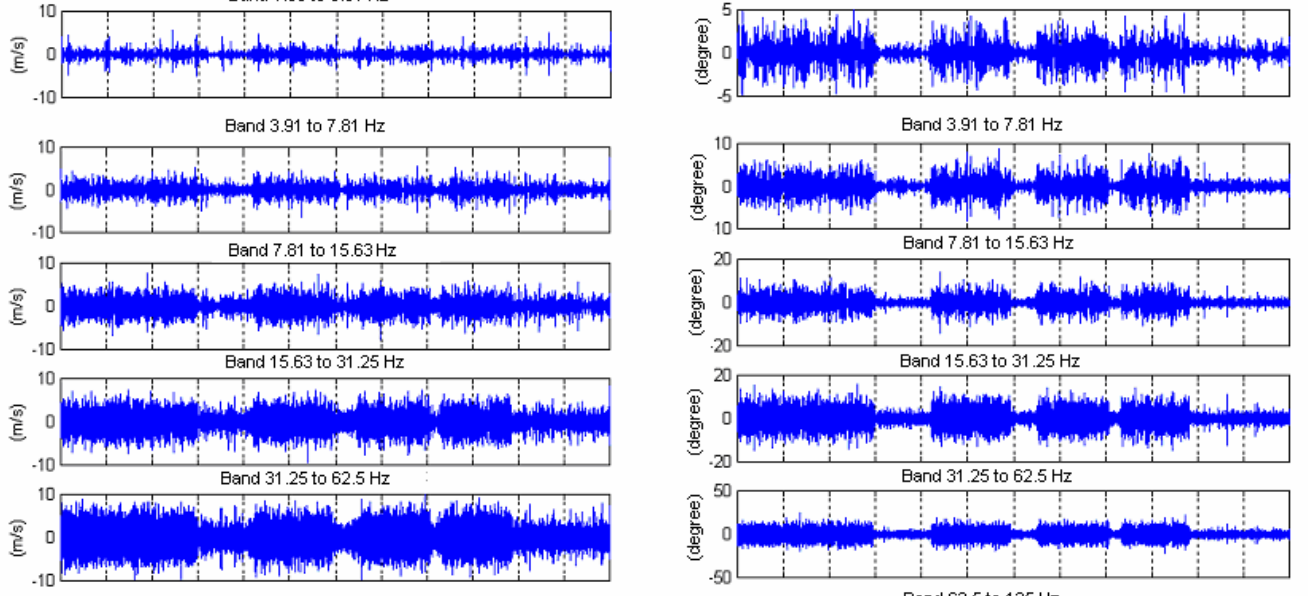

Band 62.5 to $125 \mathrm{~Hz}$
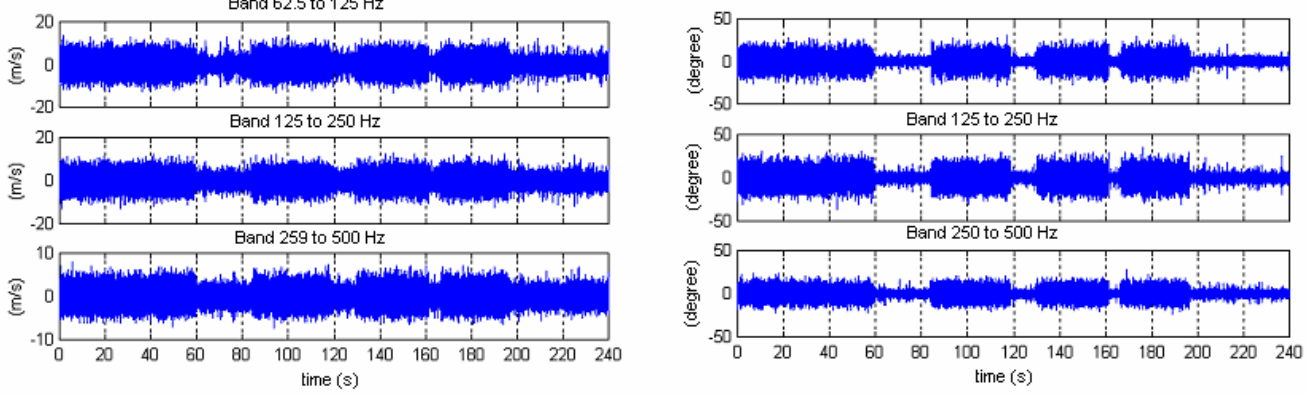

(a)

(b)

Figure 8. (a) Velocity signal decomposition ( $\mathrm{P} / \mathrm{D}=1.6)$; (b) Incident angle decomposition ( $\mathrm{P} / \mathrm{D}=1.6)$.

\section{DISCUSSION AND CONCLUSIONS}

In this work, an experimental study of the cross flow on the first rows of tube banks with square arrangement is presented. In order to detect the instabilities generated in these first rows, influencing the flow characteristics, velocity measurements had been performed in an aerodynamic channel. The tube banks used had square arrangement and P/D-ratios of 1.26, 1.4 and 1.6. For better interpretation of the results, visualizations in a water channel had been made, with a tube bank with $\mathrm{P} / \mathrm{D}=1.26$.

A phenomenon of random changes in the flow mode, characterized by the presence of transversal components to the main flow and that leads to the generation of three-dimensional structure with scale of the order of channel dimension was identified. The occurrence of such phenomenon can become an important origin of dynamic instabilities, since it can alternate the lift and drag coefficients on the tubes, modifying the dynamic response of the involved structures. The velocity measurements had been performed within the Reynolds number range called sub critical regimen, where the generated phenomena present a similar and independent behavior of the speed of the flow. The identified phenomenon is similar to the bistable flow that occurs in same geometry of two tubes located side by side to the flow. Similar phenomenon also occurs in the flow on a row of tubes, where the wake that emerge from the narrow gap between them are turned aside to form unstable sets, that can switch randomly between two flow configurations. The spurt that emerges between two cylinders interacts with the wakes formed for each cylinder, resulting that the central spurt is deflected laterally, being able to remain in this position during a time and later retaking its random behavior. The origin of this phenomenon is not completely understood. It is associated to the velocity and to the fluctuations in the neighboring wake. It is expected that, in the beginning of the process, the wakes are generated independently. If the neighboring vortices have the same phase, the fluctuation will make the 
wake to grow. If the P/D-ratio is small enough to guarantee the interaction between the wakes of the adjacent tubes, the resulting wake does not behave like on a single bluff body, due to the flow through the gap between the tubes. Preferentially, it will remain deviated behind of a tube, until a new disturbance breaks the stability and the process begins again.

In tube banks, the switching phenomenon is influenced by a highly disordered flow after the second and third row, which, in some geometries, does not allow to observe a characteristic frequency. However, the process of vortex shedding still occurs. If the interaction of the wakes, described for the two located cylinders side-by-side or a row of pipes, occurs, the change of mode will be strongly influenced by the presence of the tubes of the next row. Since no physical space for the formation of the wake is available, due to presence of the tubes of the following row, the flow will be directed for down or upwards, i. e., in the parallel direction to the axis of the tubes, resulting in a strong three-dimensional characteristic for the flow through the bank. This component, in the plan formed for the axis of the tubes and the direction of the main flow, causes a redistribution of the flow, generating transversal components to the tube axis and the flow.

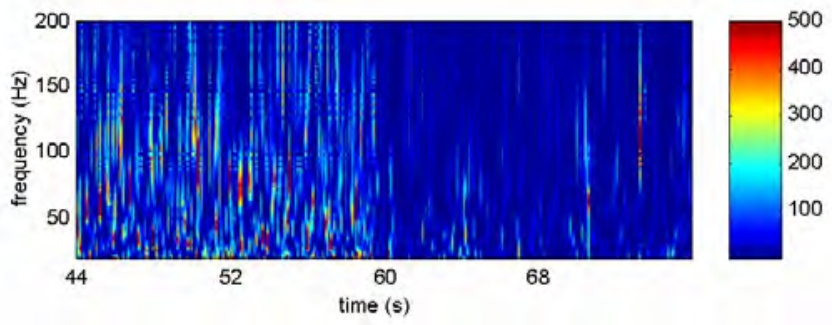

Figure 9. Spectrogram of velocity signal from $44 \mathrm{~s}$ to $76 \mathrm{~s}(\mathrm{P} / \mathrm{D}=1.6)$. (Arbitrary energy color scale)

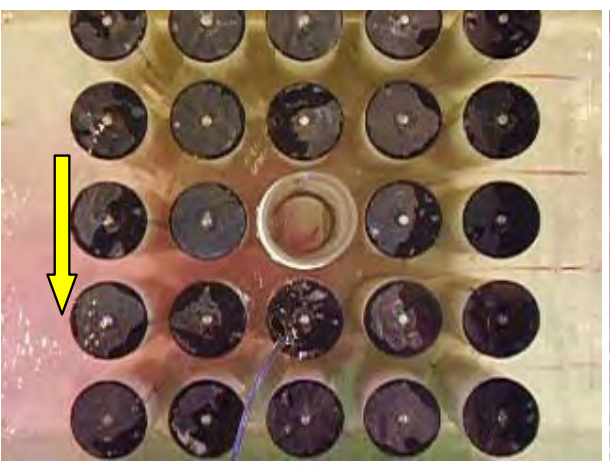

(a)

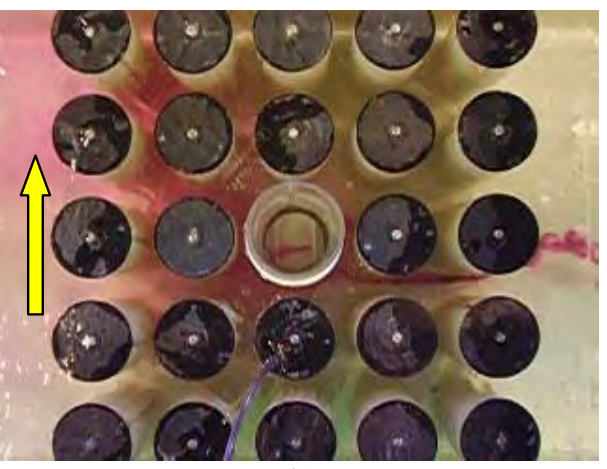

(b)

Figura 10. Flow visualization through tube bank. $\mathrm{Re}=3,07 \times 10^{4}$. (a) injection at $60 \mathrm{~mm}$ from upper wall, (b) injection at $60 \mathrm{~mm}$ from the bottom (the arrow indicate the transversal component)

Due to the acceleration/deceleration process that occurs in the spurts in the interior of the gaps in the direction of the flow, the velocity inside of the gaps of the tube bank is not homogeneously distributed. The velocity difference can cause the vortex generation and shedding in different frequencies and can be related with the instabilities that cause the three-dimensional effect. The velocity measurements, performed in the interior of the three geometries of tube banks resulted in different times of permanence between each mode, in function of the pitch ratio. For the tube bank with $\mathrm{P} / \mathrm{D}=1.26$, the time of permanence in each mode was of the order of $0.5 \mathrm{~s}$. For the bank with $\mathrm{P} / \mathrm{D}=1.4$ the mode changed within the range of 1 and $2 \mathrm{~s}$ and for the bank with $\mathrm{P} / \mathrm{D}=1.6$, was around $10 \mathrm{~s}$. This leads to conclude that the time when the change of flow mode occurs is associated with the geometry of the tube bank. The larger the P/D-ratio, the longer will be the time of permanence in each mode.

The visualization experiments had allowed recognizing that the beginning of this transversal flow occurs from the downstream region of the second row of the tube bank. In the experiments where air was the working fluid, the exchange in the flow mode occurred randomly in spontaneous form. In the experiments of visualization in water canal, such fact could not be identified. The changes in the flow mode only occurred to each new experiment, after restarting the water circulation system. Such fact also is cited in [8], where the bistable nature of the deviated flow was not detected in water channel. This could be attributed to the combination of a small degree of misalignment of the cylinders and the experimental effect, as the reasons of aspect and blockage. In [8], Authors report also that previous experiments, in which the bistable flow pattern was reported, had been carried out only in aerodynamic channels. This process, therefore, seems to be associated to the characteristics of the fluid and the channel geometric relations used in the studies. Thus, new works must be performed in order to verify, through the similarity study, if the classic dimensionless groups are able to capture all the characteristics of the studied flows. 
The performed study, that allowed the identification of the three-dimensional effect generated in the first rows of tube banks, as the transversal components to the main direction of the flow, strengthens the idea that, in being the turbulence a three-dimensional phenomenon, the two-dimensionality hypothesis, mostly used as simplification in the analysis, can not lead in account important characteristic of the flow.

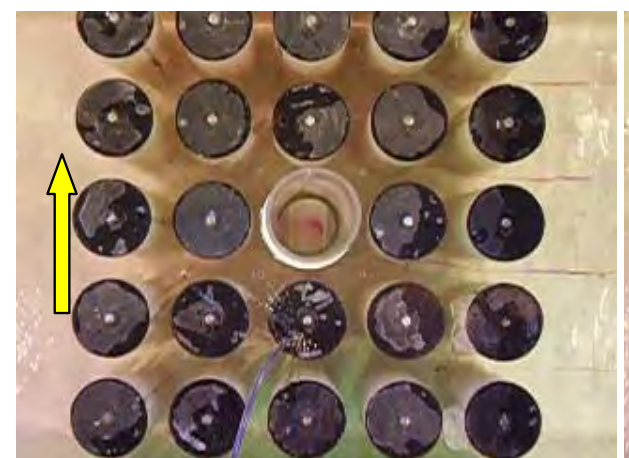

(a)

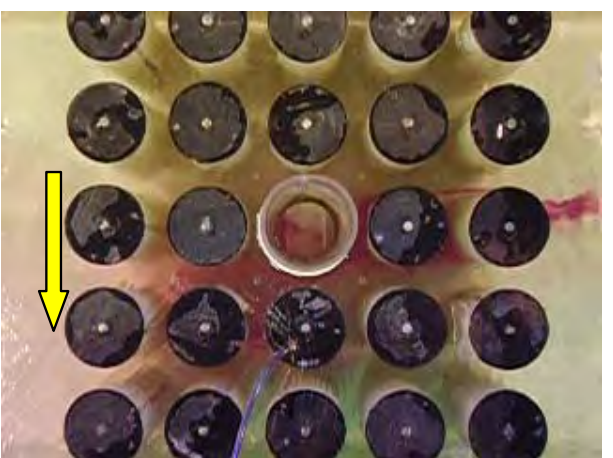

(b)

Figure 11. Flow visualization through tube bank. $\mathrm{Re}=3.18 \times 10^{4}$. (a) injection at $60 \mathrm{~mm}$ from upper wall, (b) injection at $60 \mathrm{~mm}$ from the bottom (the arrow indicate the transversal component)

\section{ACKNOWLEDGEMENTS}

Authors gratefully acknowledge the support by the CNPq - Brazilian Scientific and Technological Concil. Cláudio R. Olinto thanks CAPES, Ministry of Education, Brazil, for granting him a fellowship (PICDT).

\section{REFERENCES}

1. Blevins, R. D., Flow-Induced vibrations, $2^{\mathrm{a}}$ ed., Van Nostrand Reinhold, New York, 1990.

2. Zukauskas, A., "Heat Transfer from Tubes in Crossflow", in Advances in Heat Transfer, v. 8, Academic Press Inc., New York. 1972

3. Indrusiak, M. L. S., Goulart J. V., Olinto, C. R. and Möller, S. V., "Wavelet time-frequency analysis of accelerating and decelerating flows in a tube bank", Nuclear and Engineering Design 235, 2005, pp. 1875-1887.

4. Olinto, C. R., Indrusiak, M. L. S. Endres, L. A. M. and Möller, S. V., "Wavelet time-frequency localizations of bistable flows in banks", $18^{\text {th }}$ International Conference on Structural Mechanics in Reactor Technology (SMiRT 18), Beijing, China, 2005.

5. Olinto, C. R., Indrusiak, M. L. S. and Möller, S. V., "Experimental Study of the Bistable Flow in Tube Arrays", Journal of the Brazilian Society of Mechanics, Science and Engineering, Vol. XXVIII, No.2, 2006, pp.221-229.

6. Ziada, S, "Vorticity Sedding, Acoustic Resonance and Turbulence Excitations in Tube Bundles", Journal of the Brazilian Society of Mechanics, Science and Engineering, Vol. XXVIII, No.2, 2006.

7. Endres, L. A. and Möller, S. V., "Looking for correct dimensionless parameters for tube bank flow analysis", Journal of Fluids and Structures 15, 2001, pp. 737-750.

8. Summer, D., Wong, S. S. T., Price, S. J. and Païdoussis, M. P., "Fuid Behaviour of side-by-side circular cylinders in steady cross-flow", Journal of Fluids and Structures 13, 1999, pp. 309-338. 